$\begin{gathered}\text { EPiC Series in Built Environment } \\ \text { Volume 2, 2021, Pages 237-245 } \\ \text { ASC 2021. 57th Annual Associated Schools } \\ \text { of Construction International Conference }\end{gathered}$
Built
Environment

\title{
Challenges and Gaps in Facility Maintenance Practices
}

\author{
Mahnaz Ensafi and Walid Thabet, Ph.D. \\ Virginia Polytechnic Institute and State University \\ Blacksburg, VA
}

Facility management involves a variety of processes with a large amount of data for managing and maintaining facilities. Improved and emerging technologies available to facility managers have provided opportunities for easier access and management of the data allowing for more effective and efficient operation and maintenance. The concept of big data can help with extracting meaningful and valuable information from the available data. Current facility management practices and processes have many data management challenges, including data loss, time wasted for searching information, lack of interoperability, and so on. Lack of proper decision-making approaches and lack of maintenance planning can increase the cost of operation, influencing the quality of facility management. To effectively overcome these challenges and gaps, proper data management approaches and tools should be implemented. A structured literature review was conducted to identify challenges and gaps in three key facility processes: processing work orders, timely access to relevant data during field maintenance operations, and quality control/quality assurance of field tasks. This paper provides an overview of the three key processes, summarizes the challenges and gaps identified for each key process and proposes a framework to improve on the execution of these processes and enhance facility management decision making.

Key Words: Operation \& Maintenance, Work Orders Processing, Lifecycle Data, QA/QC, Augmented \& Mixed Reality

\section{Introduction}

Facility management (FM) is an integrated approach for operating and maintaining buildings to meet main objectives of organizations, facility managers, owners, and end users. Facility management constitute more than half of the building cost in its whole lifecycle. Operation and maintenance (O\&M) accounts for about 80 to 85 percent of capital project dollars (Thabet \& Lucas, 2017) and maintenance occupies most of the facility managers' responsibilities. Maintenance can be defined as "all the actions which aim to restore any functionality of a product within its lifecycle" (Palmarini et al., 2018, p.215). 
Facility managers are continuously under pressure to reduce costs and maximize the efficiency and productivity of staff forcing them to balance in facilities' requirements against financial limitations. Therefore, facility managers are constantly looking for new approaches for enhancing efficiency and productivity (Irizarry et al., 2013). FM involves a huge number of assets and equipment with a large amount of data associated with them. Current FM practices have challenges associated with data loss, time wasted for searching for information, lack of interoperability, and data inconsistency (Yang \& Bayapu, 2019). FM requires an approach in which there is access to accurate and reliable information about building systems as well as building components (Sadeghi et al., 2018). Lack of proper decision-making approaches and lack of a maintenance planning can increase the cost of operation from 5 to 20 percent, influencing the schedules and quality of FM (Islam et al., 2019). A reasonable management plan in addition to proper data management tools and technologies is needed to effectively utilize facility information, update the information and process, and help FM staff to reduce costs associated with O\&M. A minor change in facility management can greatly impact the cost, condition, customer satisfaction, safety and performance of the facility (Salem \& Elwakil, 2018).

As part of a broader research investigation that included all the above references, a literature review and analysis was conducted between August 2019 and November 2020 and included the ASCE and EBSCO databases. The inclusion criteria were English journals and conference papers published between 2012 and 2020. Papers focusing on facility management, BIM, and data management were searched resulting in identifying 254 relevant articles. After removing duplicates, 197 articles remained. Abstracts of these 197 articles were reviewed to remove irrelevant articles including articles focusing on computer science aspects. Upon completion of abstract reviews, 126 articles were selected, and based on the number of articles addressing the challenges as well as possible connectivity between the challenges, three major challenge areas in FM were identified: processing work orders, timely access to relevant information in the field, and proper approaches for quality assurance and quality control of field tasks. Using a second restricted search with keywords that included: work order, prioritize, augmented reality, mixed reality, quality control, and quality assurance, more papers were added to the list. After removing the studies focusing on areas other than maintenance (e.g. safety), a final list of 83 papers was left. The 83 papers were fully reviewed by the researchers. Due to the limitation of the size of this paper, the most relevant and representative references were selected resulting in a final list of 33 papers. The PRISMA guideline was used to inform the overall approach (figure 1). However, since this study is not a full systematic review, it does not fulfill all the aspects of the PRISMA guideline.

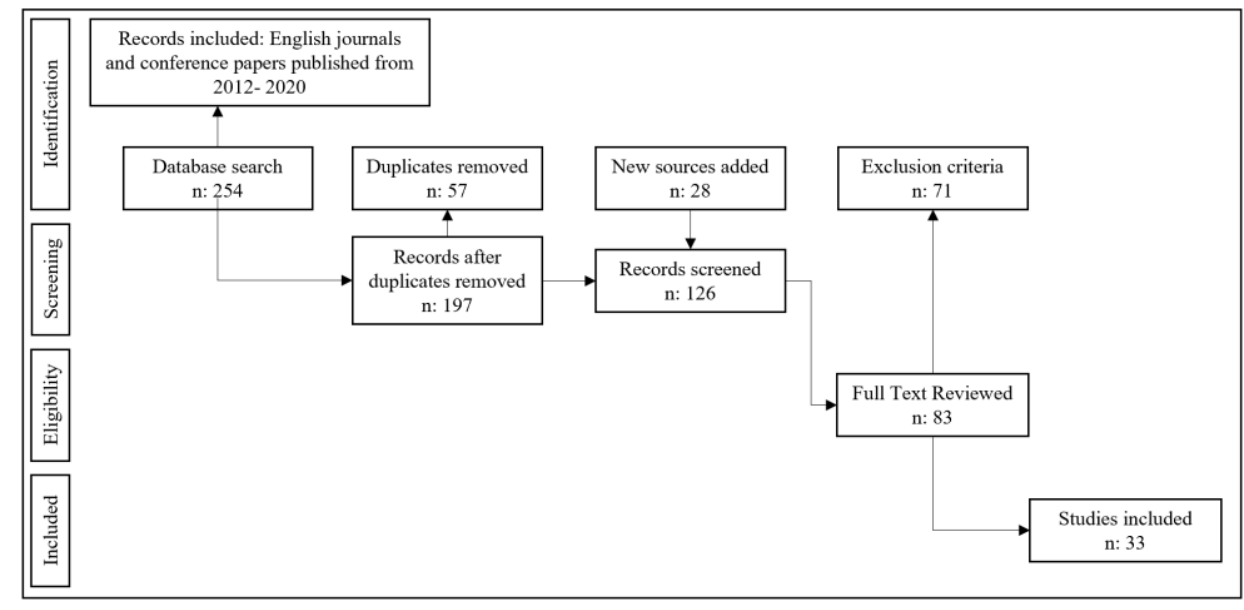

Figure 1. Search process (adapted from PRISMA) 
This paper will first provide an overview of the three challenges identified from the comprehensive literature review conducted. The paper then proposes a framework with an integrated solution to address the three challenge areas. The proposed framework uses a common database to support a unified decision-making data management approach for operation and maintenance of the facility.

\section{Challenges and Gaps with Facility Management Processes}

The following sections provides a description of the three major challenge areas with FM as identified from the literature review process.

\section{Work Order Processing}

One major challenge faced by facility managers and staff is prioritizing work orders and creating work order schedules. Work order processing is a significant part of facility management in terms of recording and reporting all maintenance tasks (Lavy et al., 2019). Large number of work orders are submitted daily, yet there are no consistent standardized approaches for performing the process. The work order processing greatly depends on staff experiences and extent of knowledge (Cao et al., 2014) as well as the number of staff available to process the orders (Beauregard \& Ayer, 2019). Delays in processing work order can lead to asset failure and downtimes impacting the cost of operation, safety and satisfaction of occupants (Salem \& Elwakil, 2018).

Identifying and having access to required data as well as critical criteria that influence work order processing will help with providing a clear and standardized approach. Besiktepe et al. (2020) identified the general criteria used for supporting maintenance decision making. They ranked the criteria to develop a multi criteria decision making model. Beauregard and Ayer (2019) used four factors of influenced group, building status, building usage, and institutional enablers to prioritize work orders for routine maintenance. Their study is focused on the relationship between facility condition and academic outcomes for K-12 education in the USA. Wang and Piao (2019) prioritized maintenance tasks for equipment components based on maintenance type and assessment of risk in terms of reliability, maintainability, economy, and detectability. Chong et al. (2019) reviewed the methods and factors used for prioritizing work orders. They categorized the factors into four groups of technical, financial, social, and political. Chen et al. (2018) explored the use of BIM combined with COBie as a geometric and semantic information repository to support FM decision making for creating maintenance work order schedules. Their proposed solution presented the existing condition of assets in the BIM model and is focused on four factors of problem type, emergency level, location, and optimal distance. Sadeghi et al. (2018) highlighted the importance of considering the source of funding and funding mechanism for maintenance decision makings in terms of capital renewal, renovation, upgrade, demolish, emergencies, etc. Salem and Elwakil (2018) determined the criticality of assets in healthcare facilities based on physical conditions, infection prevention, life safety, and revenue loss to prioritize budget allocation and reduce failure. Yang and Ergan (2017) explored required information that should be stored in BIM to automate HVAC troubleshooting. Eweda et al. (2015) created a condition assessment model considering space as the principle element for evaluation. Their model hierarchy is broken down to building type, space type, building categories, building systems, family type, and instance level. Cao, Song, and Jiang (2014) developed a framework to prioritize work orders based on feedback from both occupants and facility managers.

Based on the literature review conducted, nine factors or issues were identified that need to be considered to enhance processing of work orders. 1) Understanding and identifying information requirements will help facility managers to benefit from the systems used for FM (Yang \& Ergan, 
2017) while allowing them to create standard rules for capturing and storing work orders (Lavy et al., 2019). 2) It is important to identify assets that are more costly to operate and maintain (Islam et al., 2019). 3) Addressing the relationship between different equipment and systems in the facility in terms of their impact on each other can enhance the process (Wang \& Piao, 2019).4) Based on findings by (Besiktepe et al., 2020; Chong et al., 2019), it is better to focus on one specific type of building than generalizing a single solution to different types of facilities since the organizational objectives need to be approximately close and related. Having a well-defined organizational priority will work as a guideline for all stakeholders and remove the subjective judgements. 5) $65 \%$ of generated service requests are submitted by occupants (Mo et al., 2017). Therefore, client/occupant's satisfaction should be considered as one of the significant factors (Cao et al., 2014). 6) Not all studies have considered different types of maintenance. 7) Researchers have mainly focused on maintenance tasks completed using a single maintenance team (Chen et al., 2018). Multiple work orders completed simultaneously using multiple work crews should be considered when prioritizing and scheduling work orders.8) Budget is one of the main constraints for processing work orders and not considering it as a critical factor could prevent from providing optimal solutions. 9) Solutions proposed by previous studies are not designed in a way to allow updates and modifications required in the future.

\section{Access to Relevant Information}

There are challenges associated with identifying and filtering relevant and necessary data and making it readily available to assist FM staff while performing maintenance tasks in the field. Capturing and storing data from different information sources and in different formats create several challenges. Through the literature review, six challenges were identified. 1) There is a challenge associated with identifying the most efficient way for presenting large amounts of data to field staff (Cao et al., 2014). 2) there are difficulties with selecting the most relevant data to present to the O\&M staff for the task at hand. 3) Some information is duplicated creating data overload (Chekryzhov et al. 2018). 4)Such approaches create complexity for data exchange and retrieval, causing lack of interoperability (Martínez-Rojas et al., 2016). 5) Staff performing maintenance tasks have different levels of knowledge and experience and thus, require different levels of information (Erkoyuncu et al. (2017). 6) Providing large amount of information to the user will lead to cognitive overload impacting the user performance (Abbas et al., 2020). Poor information management can negatively impact facility management activities by creating more information than what is needed (Yang \& Ergan, 2017). This forces O\&M teams to spend more time looking for relevant information, requiring excessive processing time while distracting the team by crowding the relevant information with irrelevant data.

Abbas et al. (2020) conducted a rebar inspection task to investigate the impact of using AR systems on cognitive load and performance of users. Their findings indicated that using AR can reduce cognitive load and time while increasing performance. They also concluded that the format of information presented can impact cognitive load and thus performance. Wang and Piao (2019) implemented AR into the process of O\&M to provide information and guidance to the user. Chekryzhov et al. (2018) addressed the issue of information overload using technological equipment and proposed possible solutions leveraging AR technology. Del Amo et al. (2018) indicated that the gaps related to AR technologies have been related to one of several aspects of content-related techniques of authoring, context-awareness, or interaction analysis. Del Amo et al. (2018b) studied data visualization using AR glasses and concluded that there is a gap in having a framework for identifying required data that can be connected to the FM system. Palmarini et al. (2018) demonstrated the limitations and benefits of using AR glasses for improving maintenance performance. They highlighted the importance of understanding the best approach for adapting the information to the user considering the environment and the task. Erkoyuncu et al. (2017) proposed a 
solution addressing context awareness and authoring. They sequenced the AR content based on the equipment and user's level of expertise. Hou et al. (2015) explored the use of AR to provide instruction for assembly and their findings supported shorter duration, less errors, and lower task load. Gheisari et al. (2014) developed a system using BIM and AR mobile apps to enhance FM by having easier and faster access to the location of the components. Irizarry et al. (2013) developed a mobile AR tool to allow access to FM information and decrease data overload. Webel et al. (2013) provided two different levels of instructions on AR glasses to support different experience levels.

Although AR has been introduced to the construction industry as a possible solution to reduce information redundancy, the format of information presented as well as amount of information presented to users still require more research. Screens are often overloaded with information leading to less tendency in implementing technologies into the O\&M process (Chekryzhov et al., 2018). There exists a lack of research in automatic content contextualization. Considering context awareness can improve staff performance since the better and more accurate the information is presented, the better it will be transferred and utilized (Del Amo et al. ,2018). Additionally, it will reduce cognitive workload (Erkoyuncu et al., 2017). Maintenance staff have different levels of expertise and specific O\&M responsibilities, Also, different maintenance tasks require different information. To be more efficient, staff should have immediate access to pertinent asset information in a reasonable amount of time in the field. Also, there is a gap in having a framework for identifying, capturing, presenting, and managing required data that can be connected to the FM system (Del Amo et al., 2018b). It is significant to identify what data is needed, how it should be captured and presented (Palmarini et al., 2018), and finally how it should be edited and managed for further updates to provide a more suitable results to the user.

\section{Quality Control and Quality Assurance}

A third major challenge identified from the literature relates to tracking and improving quality assurance (QA) and control (QC) of field tasks. Traditional QA/QC is based on collecting data and reports and manually entering the information into the FM system. Such processes require a significant amount of time and cost which impacts the QA/QC process. Additionally, shortage of labor or experienced staff can always result in challenges for the FM. Staff performance should always be analyzed and evaluated to identify gaps, modify the tools and training provided and enhance performance (del Amo et al., 2018b). Actively monitoring and analyzing the accuracy of maintenance steps are significantly important. Although many studies have considered the significance of QA/QC during construction more work is still needed to improve QA/QC during facility operations and maintenance.

Chalhoub et al. (2018) used mixed reality for construction QA/QC by comparing the 3D model with the actual building on-site. del Amo et al. (2018b) studied the integration of AR into the maintenance process and they proposed a framework describing information type and formats as well as interaction modes for improving the efficiency of using AR for maintenance. Karji et al. (2017) reviewed the previous studies on integration of BIM, AR, and image processing in construction industry. They believe that image processing can be used to analyze productivity and perform QA. Liu and Zettersten (2016) highlighted the importance of having quality check. Martínez-Rojas et al. (2016) investigated the advantages of using information and communication technologies and their benefits for addressing quality control, progress monitoring, and costs. Westerfield et al. (2015) applied the use of AR into the assembly process by providing real-time feedbacks about the procedure. Fiorentino et al. (2014) used AR for presenting maintenance instructions and their results showed up to $92.4 \%$ error reduction and up to $79 \%$ time reduction. Kasprzak et al. (2013) conducted a survey and concluded that there is a gap in having enough QA/QC mechanisms for maintenance process standardization. 
As discussed above, there is a need for controlling and analyzing the accuracy of the steps taken for training and maintenance. Although providing step by step instructions can reduce errors and asset failures, training and instructions should be continuously updated based on maintenance performance as well as operator level of experience and knowledge. Researchers have highlighted the importance of automation in documentation and progress checking to reduce the time and staff required (Fiorentino et al., 2014). While AR glasses are used for remote collaboration to provide guidance and feedback, they can also allow QA/QC. Many available AR devices are able to recognize users' gesture (Cheng et al., 2020) and therefore, the interaction between user and content can be captured and analyzed (del Amo et al., 2018). Use of multimodal interaction methods have been suggested by previous studies to track the interaction with the objects to record time of maintenance as well as staff performance (del Amo et al., 2018b). Capturing movements can help to identify errors at the time of performance. Furthermore, due to the high number of maintenance tasks, not all the performed tasks are monitored and checked. With automated QA/QC, most of the tasks can be checked. Such strategies can help different facility managers to avoid double-checking and processing. It can also reduce their challenges by balancing the requirements of the facility with financial limitations.

\section{Proposed Framework for Addressing the Challenges}

Considering the three challenge areas discussed above, large amount of data is generated for various assets and is collected by different facility management systems. Therefore, there is a need for a common database to collect and store asset information (Farghaly et al., 2018) to allow connecting different facility management tasks and increase interoperability. Also, it is important to consider a continuous data collection within each task and continuous data collection moving from one task to the other. There is a need for identifying information requirements in terms of what data to collect at each step as well as data types and formats in order to increase consistency in data collection and exchange. Such approach will allow to benefit from informed and data-driven O\&M decision making.

Figure 2 shows a proposed abstract theoretical framework defining a workflow that integrates three processes: work order processing, access and retrieval of critical and relevant maintenance data, and QA/QC using a common database to allow for a data-driven decision-making approach and increase data interoperability. When work orders are requested, information from service requests will be used by the system to collect required data from the common database (part 1), identify the issue, and prioritize maintenance tasks based on defined criteria. The maintenance schedule will then be generated. The maintenance manager can modify the schedule order generated and provide new input into the database (part 5). Based on the final schedule generated, the responsible crews will then be notified of the maintenance tasks assigned to them (part 2). Augmented/Mixed reality (AR/MR) glasses can be utilized to provide location of each task and relevant information required. Level of detail of information, structure and display format should be defined based on the asset type, nature of the task performed, and user preferences to reduce cognitive overload and enhance performance. Any missing information not provided to the user through the glasses can be retrieved by the user directly from the database using a built-in search and capture feature. The framework will be developed to learn and automatically capture changes to update the information provided in future tasks.

AR/MR glasses can be used to provide a step-by-step instruction based on the level of knowledge and expertise of the assigned crew to perform the maintenance task (part 3). Instructions can be provided using an application on the glasses or through remote collaboration with other users. Information provided through the glasses is accessed from the central common database (part 1). 


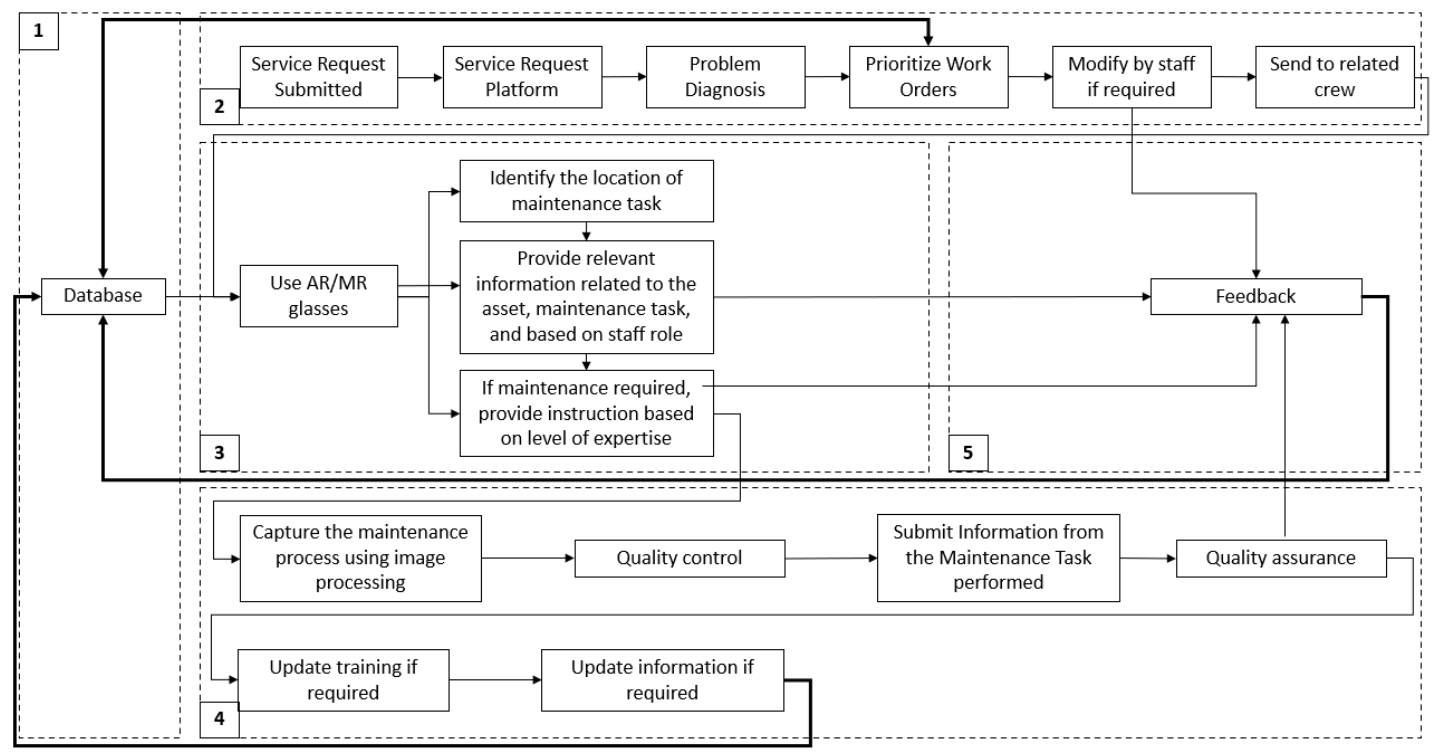

Figure 2. Framework for connecting the three challenge areas

While the AR/MR glasses provide access to needed information, they can also be used for QA/QC. If the guidance is provided to field staff using remote collaboration, users can receive feedback regarding their performance directly through their supervisor during and after the maintenance task is complete. If the guidance is provided through system instructions or videos, the glasses can use images processing to capture and analyze the user's gesture to provide feedback. Additionally, with the ability to record a maintenance session, recorded performance can be used for future quality check if needed. The proposed framework can address a more comprehensive QA/QC while reducing the time and staff required for performing the QA/QC. Feedback from $\mathrm{QA} / \mathrm{QC}$ can be used to update training requirements, as well as instructions provided to field personnel to increase their performance (part $1 \& 4$ ). Information collected over time and through these processes can be used to support datadriven decision-making since the information are consistently and continuously collected and analyzed to find patterns and update the system accordingly. Additionally, such approach can avoid data duplication by using the data collected in one process for another process.

\section{Conclusion}

Due to the rapid improvements and affordability of technologies used for FM, more complicated and larger amount of data are created and are becoming more available to facility managers (Cao et al., 2014). The concept of big data can help with extracting meaningful and valuable information from the available data (Yang \& Bayapu, 2019). Additionally, data analytics methodologies and machine learning algorithms can be used to process and investigate the collected data, find patterns, and drive conclusions to benefit the facilities and support their decision makings (Assaf et al., 2020).

The authors are planning to focus on the first area, processing work orders, moving forward with this research since this process involves a variety of factors and rankings. Therefore, implementing datadriven decision making and machine learning can assist with performing the process while supporting consistency in collecting and storing data. The authors are planning to conduct more semi structured interviews in addition to surveys to collect valuable knowledge to propose a solution for processing 
work orders. The solution will be validated using case studies and industry partners involved in facility maintenance.

Although many studies have highlighted the importance of consistency and interoperability in FM, the processes used in facilities are still lacking a proper connected framework and data management to support informed and data- driven decision making. This paper provided an overview of prior research focused on three challenge areas and proposed a theoretical framework to address them. It is believed that the framework can enhance performance and quality of FM while reducing costs.

\section{References}

Abbas, A., Seo, J., \& Kim, M. (2020). Impact of Mobile Augmented Reality System on Cognitive Behavior and Performance during Rebar Inspection Tasks. Journal of Computing in Civil Engineering, 34(6), 04020050.

Beauregard, M. A., \& Ayer, S. K. (2019). Leveraging previously reported research to create a decision support tool for institutional facility maintenance. Journal of Facilities Management.

Besiktepe, D., Ozbek, M. E., \& Atadero, R. A. (2020). Identification of the Criteria for Building Maintenance Decisions in Facility Management: First Step to Developing a Multi-Criteria Decision-Making Approach. Buildings, 10(9), 166.

Cao, Y., Wang, T., \& Song, X. (2015). An energy-aware, agent-based maintenance-scheduling framework to improve occupant satisfaction. Automation in Construction, 60, 49-57.

Chalhoub, J., Alsafouri, S., \& Ayer, S. K. (2018, January). Leveraging site survey points for mixed reality BIM visualization. In Construction Research Congress 2018 (pp. 326-335).

Chekryzhov V., Kovalev I. A., \& Grigoriev A. S. (2018). An approach to technological equipment performance information visualization system construction using augmented reality technology. In MATEC Web of Conferences (Vol. 224, p. 02093). EDP Sciences.

Chen, W., Chen, K., Cheng, J. C. P., Wang, Q., \& Gan, V. J. L. (2018). BIM-based framework for automatic scheduling of facility maintenance work orders. Automation in Construction, 91, 15-30.

Cheng, J. C., Chen, K., \& Chen, W. (2020). State-of-the-art review on mixed reality applications in the AECO industry. Journal of Construction Engineering and Management, 146(2), 03119009.

Chong, A. K. W., Mohammed, A. H., Abdullah, M. N., \& Rahman, M. S. A. (2019). Maintenance prioritization-a review on factors and methods. Journal of Facilities Management.

del Amo, I. F., Erkoyuncu, J. A., Roy, R., Palmarini, R., \& Onoufriou, D. (2018a). A systematic review of Augmented Reality content-related techniques for knowledge transfer in maintenance applications. Computers in Industry, 103, 47-71.

del Amo, I. F., Erkoyuncu, J. A., Roy, R., \& Wilding, S. (2018b). Augmented Reality in Maintenance: An information-centred design framework. Procedia Manufacturing, 19, 148-155.

Erkoyuncu, J. A., del Amo, I. F., Dalle Mura, M., Roy, R., \& Dini, G. (2017). Improving efficiency of industrial maintenance with context aware adaptive authoring in augmented reality. Cirp Annals, 66(1), 465-468.

Eweda, A., Zayed, T., \& Alkass, S. (2015). Space-based condition assessment model for buildings: Case study of educational buildings. Journal of Performance of Constructed Facilities, 29(1), 04014032.

Farghaly, K., Abanda, F. H., Vidalakis, C., \& Wood, G. (2018). Taxonomy for BIM and asset management semantic interoperability. Journal of Management in Engineering, 34(4), 04018012. 
Fiorentino, M., Uva, A. E., Gattullo, M., Debernardis, S., \& Monno, G. (2014). Augmented reality on large screen for interactive maintenance instructions. Computers in Industry, 65(2), 270-278.

Gheisari, M., Williams, G., Walker, B. N., \& Irizarry, J. (2014). Locating building components in a facility using augmented reality vs. paper-based methods: A user-centered experimental comparison. In Computing in Civil and Building Engineering (2014) (pp. 850-857).

Hou, L., Wang, X., \& Truijens, M. (2015). Using augmented reality to facilitate piping assembly: an experiment-based evaluation. Journal of Computing in Civil Engineering, 29(1), 05014007.

Irizarry, J., Gheisari, M., Williams, G., \& Walker, B. N. (2013). InfoSPOT: A mobile Augmented Reality method for accessing building information through a situation awareness approach. Automation in Construction, 33, 11-23.

Islam, R., Nazifa, T. H., \& Mohamed, S. F. (2019). Factors influencing facilities management cost performance in building projects. Journal of Performance of Constructed Facilities, 33(3), 04019036.

Karji, A., Woldesenbet, A., \& Rokooei, S. (2017). Integration of Augmented Reality, Building Information Modeling, and Image Processing in Construction Management: A Content Analysis. In AEI 2017 (pp. 983-992).

Kasprzak, C., Ramesh, A., \& Dubler, C. (2013). Developing standards to assess the quality of BIM criteria for facilities management. In AEI 2013: Building Solutions for Architectural Engineering (pp. 680-690).

Lavy, S., Saxena, N., \& Dixit, M. (2019). Effects of BIM and COBie Database Facility Management on Work Order Processing Times: Case Study. Journal of Performance of Constructed Facilities, 33(6), 04019069.

Liu, R., \& Zettersten, G. (2016). Facility sustainment management system automated population from building information models. In Construction Research Congress 2016 (pp. 2403-2410).

Martínez-Rojas, M., Marín, N., \& Vila, M. A. (2016). The role of information technologies to address data handling in construction project management. Journal of Computing in Civil Engineering, 30(4), 04015064.

Mo, Y., Zhao, D., Syal, M., \& Aziz, A. (2017). Construction work plan prediction for facility management using text mining. In Computing in Civil Engineering 2017 (pp. 92-100).

Palmarini, R., Erkoyuncu, J. A., Roy, R., \& Torabmostaedi, H. (2018). A systematic review of augmented reality applications in maintenance. Robotics and Computer-Integrated Manufacturing, 49, 215-228.

Salem, D., \& Elwakil, E. (2018). Develop an Assessment Model for Healthcare Facilities: A Framework to Prioritize the Asset Criticality for the Capital Renewals. ICCREM 2018, 8288.

Thabet, W., \& Lucas, J. (2017). Asset data handover for a large educational institution: Case-study approach. Journal of Construction Engineering and Management, 143(11), 05017017.

Wang, T. K., \& Piao, Y. (2019). Development of BIM-AR-Based Facility Risk Assessment and Maintenance System. Journal of Performance of Constructed Facilities, 33(6), 04019068.

Webel, S., Bockholt, U., Engelke, T., Gavish, N., Olbrich, M., \& Preusche, C. (2013). An augmented reality training platform for assembly and maintenance skills. Robotics and autonomous systems, 61(4), 398-403.

Westerfield, G., Mitrovic, A., \& Billinghurst, M. (2015). Intelligent augmented reality training for motherboard assembly. International Journal of Artificial Intelligence in Education, 25(1), 157-172.

Yang, E., \& Bayapu, I. (2019). Big Data analytics and facilities management: a case study. Facilities,268-281.

Yang, X., \& Ergan, S. (2017). BIM for FM: information requirements to support HVAC-related corrective maintenance. Journal of Architectural Engineering, 23(4), 04017023. 